\title{
Ankyrins in human health and disease - an update of recent experimental findings
}

Damian B. Chagula ${ }^{1}$, Tomasz Rechciński ${ }^{2}$ Karolina Rudnicka ${ }^{1}$, Magdalena Chmiela ${ }^{1}$

\begin{abstract}
${ }^{1}$ Laboratory of Gastroimmunology, Department of Immunology and Infectious Biology, Faculty of Biology and Environmental Protection, University of Lodz, Lodz, Poland ${ }^{2}$ Department of Cardiology, Bieganski Regional Speciality Hospital, Medical University of Lodz, Lodz, Poland
\end{abstract}

Submitted: 12 October 2017

Accepted: 25 February 2018

Arch Med Sci 2020; 16 (4): 715-726

DOI: https://doi.org/10.5114/aoms.2019.89836

Copyright $\odot 2018$ Termedia \& Banach

\section{Abstract}

Ankyrins are adaptor molecules that in eukaryotic cells form complexes with ion channel proteins, cell adhesion and signalling molecules and components of the cytoskeleton. They play a pivotal role as scaffolding proteins, in the structural anchoring to the muscle membrane, in muscle development, neurogenesis and synapse formation. Dysfunction of ankyrins is implicated in numerous diseases such as hereditary spherocytosis, neurodegeneration of Purkinje cells, cardiac arrhythmia, Brugada syndrome, bipolar disorders and schizophrenia, congenital myopathies and congenital heart disease as well as cancers. Detecting either down- or over-expression of ankyrins and ergo their use as biomarkers can provide a new paradigm in the diagnosis of these diseases. This paper provides an outline of knowledge about the structure of ankyrins, and by making use of recent experimental research studies critically discusses their role in several health disorders. Moreover, therapeutic options utilizing engineered ankyrins, designed ankyrin repeat proteins (DARPins), are discussed.

Key words: ankyrin-related diseases, DARPins, adaptor proteins.

\section{Introduction}

\section{Ankyrin proteins}

Ankyrins (ANK) are the most expressed group of modular proteins present in numerous if not all metazoan cells, but absent in other life forms such as plants, fungi and prokaryotes [1]. However, recently five ANK proteins of Orf virus (ORFV) have been identified as possible substrates of asparaginyl hydroxylase, which downregulates the hypoxia-inducible factor (HIF). This is a new mechanism used by viruses to de-repress HIF and develop infection. However, the possible influence of the ORFV ANKHIF interaction on viral replication has yet to be explored [2]. The numerous genes encoding ANK-containing proteins present in the genome of various pathogenic microbial agents have been acquired from eukaryotic organisms due to horizontal gene transfer [3]. The major function of ANK is to organize the physiological assembly of membrane proteins $[1,4]$ to actin [5-7], and spectrin-based membranes $[6,8,9]$. These membrane proteins include the voltage-gated sodium channels, sodium potassium adenosine triphosphatase, anion and cation exchangers as

\author{
Corresponding author: \\ Prof. Magdalena Chmiela \\ Laboratory \\ of Gastroimmunology \\ Department of Immmunology \\ and Infectious Biology \\ Faculty of Biology \\ and Environmental Protection \\ University of Lodz \\ 12/16 Banacha St \\ 90-237 Lodz, Poland \\ E-mail: magdalena.chmiela@ \\ biol.uni.lodz.pl
}




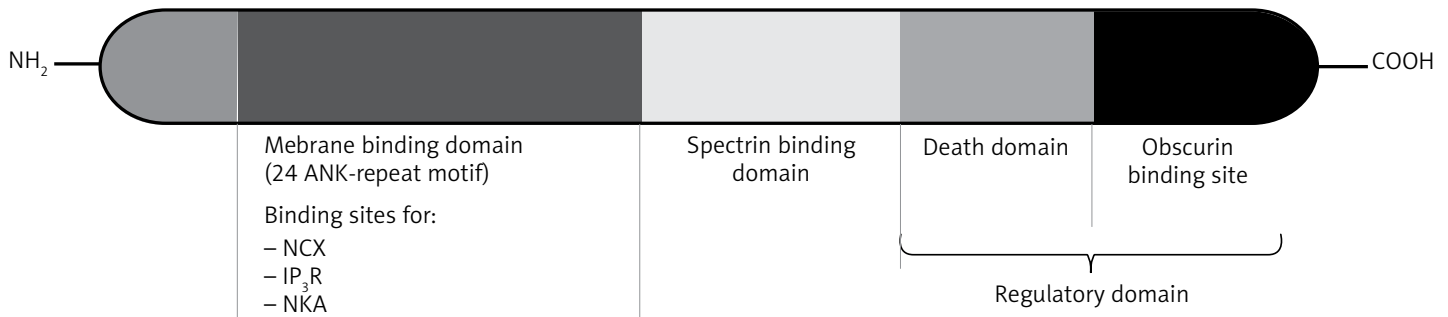

$-\mathrm{KCNQ} 2 / 3$

- VGSCS

- L1-CAMs

Figure 1. Ankyrin (ANK) domains and their ligands. The membrane binding domain binds to sodium calcium exchanger (NCX), inositol 1,4,5-triphosphate receptors $\left(I_{3} R\right)$, sodium potassium adenosine triphosphatase (NKA), potassium voltage gated channel subunit 2/3 (KCNQ 2/3), voltage-gated sodium channels (VGSCs) as well as L1-cell adhesion molecules (L1-CAMs) via the ankyrin repeat motif. The ankyrins also interact with the actin-based cytoskeleton membrane via the B-spectrin domain. The regulatory domain modulates the interaction of both membrane binding and spectrin binding domains ([5, 7], modified)

well as the L1-cell adhesion molecule family [1, $5,10]$. As a result of alternative splicing of three genes, an assorted group of ANK polypeptides have emerged: ANK R encoded by the ank1 gene predominant in the red blood cells [10], ANK B encoded by ank2 [1, 5, 11] abundantly expressed in the nervous system $[4,11]$ and ANK G encoded by ank3 [12, 13] expressed in most human tissues $[1,10]$. Organisms such as Caenorhabditis elegans (nematode worm) and Drosophila melanogaster (fruit fly) possess one and two ANK genes [1] respectively, whereas humans (and other vertebral organisms) express three ANK genes. This variation is probably due to genome duplications [12]. Virtually nothing is known about the relationship between ANK co-expressed in the same tissue, so the loss of function in ANK as ascribed to their interaction is a subject for further investigation [8]. Structurally, typical ANK proteins are made up of conserved and specialized domains (Figure 1); these include the ANK repeats, a spectrin-binding domain, and a regulatory domain containing a death domain and an obscurin binding site [4, 5]. Recently, ANK have emerged as multifunctional adaptor proteins $[1,8,13,14]$, which, due to their structural make up and interaction with other proteins as aforementioned, have been suggested to play pivotal roles as scaffolding proteins [1] in the structural anchoring to the muscle membrane [14], in muscle development [10], neurogenesis and synapse formation $[11,15,16]$. Regulatory properties of ANK are linked to ubiquitin-mediated degradation of proteins: a critical process for proper cell functioning [17]. The dysfunction of ANK is implicated in numerous diseases, some of which are hereditary spherocytosis, neurodegeneration of Purkinje cells $[1,9]$, some cardiac arrhythmias [1, 5, 10, 18], Brugada syndrome, bipolar disorders and schizophrenia [11, 13, 19]. Additionally, congenital myopathies and congenital heart disease [15], as well as cancers, have been reported, as a result of the disruption in not only ANK proteins but also proteins containing ANK repeats, e.g. muscle ANK repeat containing proteins (MARPs) [14, 20, 21] and Notch proteins. Recently it has been shown that ANK repeat proteins can express their biological function not only due to specific protein-protein interactions [22] but also due to protein-lipid [23] and protein-sugar interactions [24].

\section{Ankyrin domains \\ Membrane binding domain}

Instigating at the $\mathrm{N}$-terminal of the ANK gene $[4,12]$, membrane binding domain (MBD) is made of 24 tandem ANK repeats [1, 10, 12], which interact with L1-cell adhesion molecules, inositol 1,4,5-triphosphate receptors, as well as ion channels and pumps, which include sodium potassium adenosine triphosphatase, sodium calcium exchangers, voltage-gated sodium channels, and potassium voltage gated channel subunit $2 / 3$ [7, 8, 11, 25-28]. Ankyrins repeats are made up of 33 amino acid segments, each of which forms two antiparallel $\alpha$ helices and a $\beta$-hairpin $[1,4,20]$.

\section{$\beta$-spectrin binding domain}

Located centrally [4], the $\beta$-spectrin binding domain (BSD) connects the actin cytoskeleton to the ANK. This is important for erythrocytes' normal structural function; hence the conjoint expression of both ANK and spectrin is crucial for appropriate physiology and function of neuronal and epithelial membranes $[1,10]$.

\section{Regulatory domain}

The regulatory domain is made up of a death and C-terminal domains [4, 10], and it is known to interact with obscurin, tumour necrosis factor receptor superfamily (Fas) members and human 
Table I. Types of ankyrins (ANK) and their isoforms according to molecular weight and localization ([8], modified)

\begin{tabular}{|lcccccc|}
\hline \multirow{2}{*}{ ANK isoforms } & \multicolumn{5}{c|}{ Organ/cell/tissue } \\
\cline { 2 - 7 } & Brain & Heart & Skeletal muscle & Lung & Kidney & Erythrocyte \\
\hline ANK B & $220 \mathrm{kDa}$ & $160 \mathrm{kDa}$ & $220 \mathrm{kDa}$ & $220 \mathrm{kDa}$ & $220 \mathrm{kDa}$ & - \\
& $440 \mathrm{kDa}$ & $220 \mathrm{kDa}$ & & & & \\
\hline ANK G & $270 \mathrm{kDa}$ & $190 \mathrm{kDa}$ & $107-130 \mathrm{kDa}$ & $190 \mathrm{kDa}$ & $119 \mathrm{kDa}$ & - \\
& $480 \mathrm{kDa}$ & & $119 \mathrm{kDa}$ & $200-215 \mathrm{kDa}$ & $190 \mathrm{kDa}$ & \\
& & & & & $200-215 \mathrm{kDa}$ & \\
\hline ANK R & $186 \mathrm{kDa}$ & $210 \mathrm{kDa}$ & $25 \mathrm{kDa}$ & - & - & $186 \mathrm{kDa}$ \\
& $215 \mathrm{kDa}$ & & & & & $215 \mathrm{kDa}$ \\
\hline
\end{tabular}

A

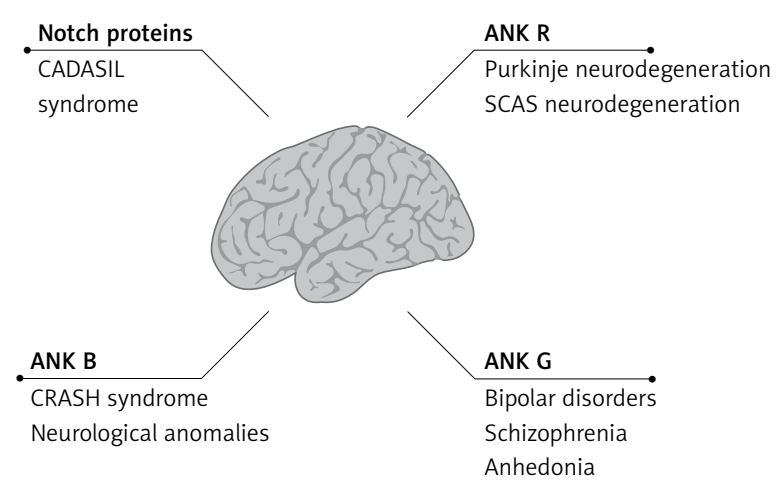

B

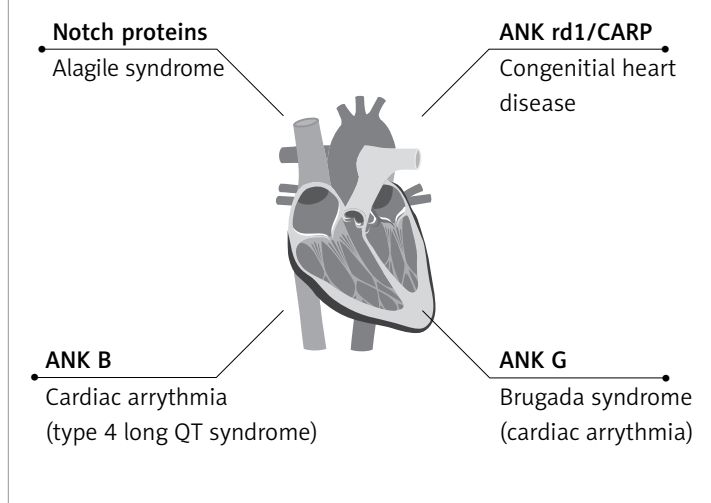

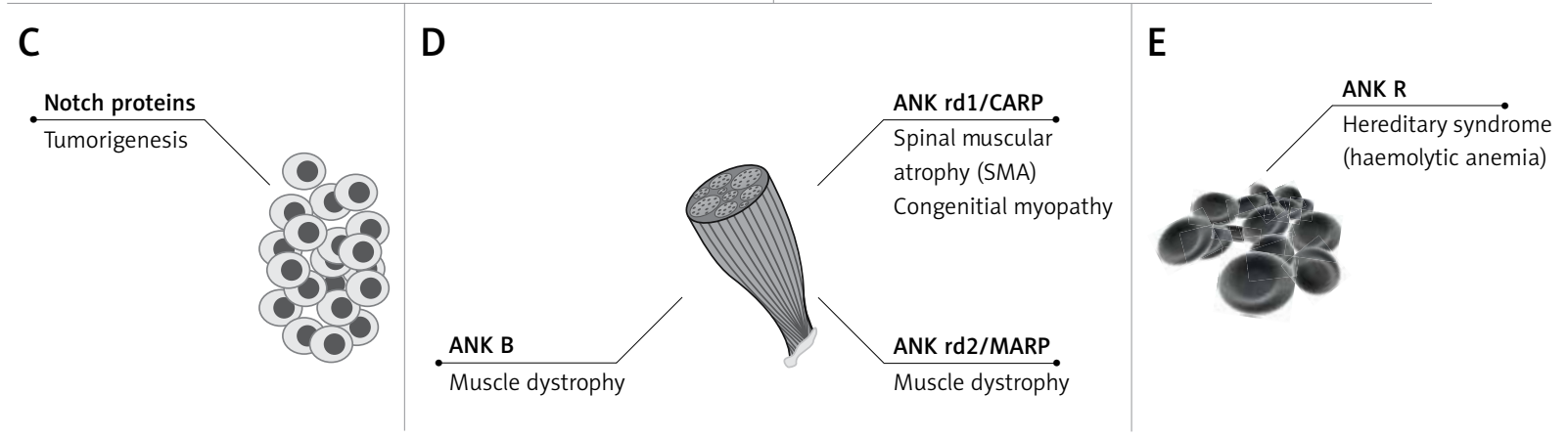

ANK - ankyrin, CRASH - corpus callosum hypoplasia, retardation adducted thumbs spastic paraplegia, hydrocephalus, SCAS - spinocerebrospinal ataxia type syndrome, CADASIL - cerebral autosomal dominant arteriopathy with subcortical infarcts and leukoencephalopathy, Ankrd1/CARP - ankyrin repeat domain 1 proteins/cardiac ankyrin repeat protein, MARP - muscle ankyrin repeat protein.

Figure 2. The involvement of ankyrins and ankyrin repeat domain containing proteins in human diseases affecting: A - central nervous system, B - heart, C - various host cells, D - skeletal muscles and E - erythrocytes

DnaJ protein containing J-domain 1 (Hdj-1). It also modulates the binding of proteins to both MBD and $\beta$-spectrin domains [4]. Despite the unknown functions, the death domains are the most highly conserved ANK domains [1].

\section{Types of ankyrins and associated diseases}

Three types of ANK - ANK R, ANK B and ANK G - may occur in different isoforms, which are tissue specific or distributed within several organs, which may suggest that they exhibit different functions (Table I). The additive or co-regulatory effects of various ANK and their forms in conjunction with tissue localization has been suggested [8]. Moreover, ANK mutations or networking proteins provide several disease phenotypes associated with ANK dysfunction in erythrocytes, heart, central nervous system and skeletal muscles (Figure 2).

\section{Ankyrin R, hereditary spherocytosis, Purkinje neurodegeneration and SCA5}

Encoded by the ank1 gene $[1,5,10]$ located on human chromosome $8 p 11[8,10,29]$, ANK R is the only ANK expressed in erythrocytes [10], also 
expressed in neurons, myocardium and skeletal muscles in different isoforms [1, 14]. Depending on the isoform, they vary from a molecular weight of 25 to $215 \mathrm{kDa}$ (Table I). Ankyrin together with spectrin are essential for supporting the integrity of structural membranes in erythrocytes $[8,9]$. Unsurprisingly, mutations in ank $1[1,10]$ and defective proteins such as spectrin [9] are associated with a serious erythrocyte membrane disorder - hereditary spherocytosis (HS) $[1,8,10]$. This haemolytic anaemia [29] is characterized by an increased reticulocytes, sickle-celled anaemia [8], icterus and splenomegaly [29,30]. These mutations lead to loss of ANK and spectrin proteins, consequently hampering the mechanical pliability of the red blood cells' membrane $[1,10]$. Defective spherical-like erythrocytes are eliminated in the spleen, hence the haemolysis associated with HS $[29,30]$. Bennet and Healy also underscored ANK R protein's importance in the stability of the Rh complex, which when absent can lead to anaemia [1]. The binding of ANK to the Rh complex is a prerequisite for the latter's proper expression in the red blood cells. Similarly, Rh null erythrocytes were shown to have features of spherocytosis, confirming that the down-expression of ANK R is linked with HS.

Ankyrin $R$ dysfunction has also been associated with Purkinje neurodegeneration [9]. Located excessively in the cerebellum, ANK R localizes to the cerebellar Purkinje and granule cells. It was demonstrated in mice normoblastosis models that the levels of ANK R protein were hampered in the neuronal cells, including Purkinje cells due to two spinocerebellar ataxia type 5 linked mutations of $\beta$-III spectrin. On the other hand, mice $(\mathrm{nb} / \mathrm{nb})$ deficient in the ank 1 gene developed severe haemolytic anaemia but also ataxia, unusual tremors and the loss of about half of Purkinje neuronal cells. Moreover, slow neurodegeneration and motor deficit ensue from this loss of cells. Furthermore, Clarkson et al. demonstrated on animal models that in normal mice, the $\beta$-III spectrin/ANK R complex elevates sodium levels and neuronal activity [9]. Conversely, the mutant $\beta$-III spectrin/ANK complexes failed to do so. Consequently, in the latter case, the ability to form stable complexes between ANK R and its interacting partners in the Purkinje cells is disrupted, suggesting this as a major mechanism by which mutants of $\beta$-III spectrin cause ataxia. A point to note is that although ANK $R$ is also present in the skeletal muscles, muscle dystrophy has not been reported to be linked with its dysfunction [8].

\section{Ankyrin B, cardiac arrhythmia and CRASH syndrome}

Ankyrins, especially ANK B and spectrins, are related to electrical and structural forms of heart diseases [18]. Recently ANK as well as cardiolipin-1, adipokines, galectin-3 and vitamin deficiency have been considered potential biomarkers of cardiovascular diseases [31-33], in addition to standard risk factors such as hypercholesterolaemia [34]. These biomarkers are supposed to be crucial to apply the best therapy in heart failure and predicting the risk of cardiovascular development [35].

Encoded by the ank2 gene $[1,5,10]$ found in human chromosome $4 q 25-27$, ANK $B$ has been found in the heart, brain, skeletal muscles and thymus [1]. The most common forms of ANK B are the 220 and $440 \mathrm{kDa}$ isoforms (Table I). Aberrations in the ank 2 gene are directly related to anomalies in the cardiovascular physiology $[1,5]$, leading to cardiac arrhythmia (also called ANK B syndrome) $[1,10]$. Owing to the even distribution of ANK B protein, that is, in the atria and ventricles of the heart, as well as in the sinoatrial node (pacemaker), not surprisingly, this type of cardiac arrhythmia (which also used to be known as type 4 long QT syndrome) [10], is characterized by an irregular pulse due to atrial fibrillation being too slow (bradycardia) or catecholaminergic polymorphic tachycardia, idiopathic ventricular fibrillation [8, 36-38], sick sinus syndrome, and even sudden cardiac death $[1,8,10]$. A missense was spotted in the exon utilizing DNA sequencing techniques, bringing about the substitution of glycine by glutamic acid. Loss of most functions of the ank 2 variants at the $C$-terminal domain has been directly linked with a broad spectrum of clinical phenotypes for cardiac arrhythmia. Ankyrin B is indispensable in targeting and maintenance of ion channels and transporters [10], modulating $\mathrm{Ca}^{2+}$ dynamics at the sarcoplasmic reticulum and T-tubule junction membranes of the cells of heart ventricles $[1,5,8,10]$. It has been posited that the flux of calcium ions is facilitated by ANK B complex from the sarcoplasmic reticulum via inositol 1,4,5-triphosphate receptors and across the plasma membrane via the sodium calcium exchangers (NCX) and sodium potassium adenosine triphosphatase (NKA) [12] (Figure 3). This was confirmed using mouse models, whereas mice with only one functional ank2 gene exhibited reduced expression of NCX, NKA and IP3 receptor [8, 10]. Additionally to this reduced expression, it was demonstrated using ank2-heterozygous mice models that their sinoatrial node (SAN) cells exhibited inappropriate targeting of CaV1.3, a key modulator of extracellular $\mathrm{Ca}^{2+}$ entry necessary for proper pacemaker function [8]. In spite of the so far understood roles of ANK B in normal heart functioning, virtually nothing is known pertaining to the modulation of ank 2 transcription and translation in the heart [5]. Recently it has been demonstrated 


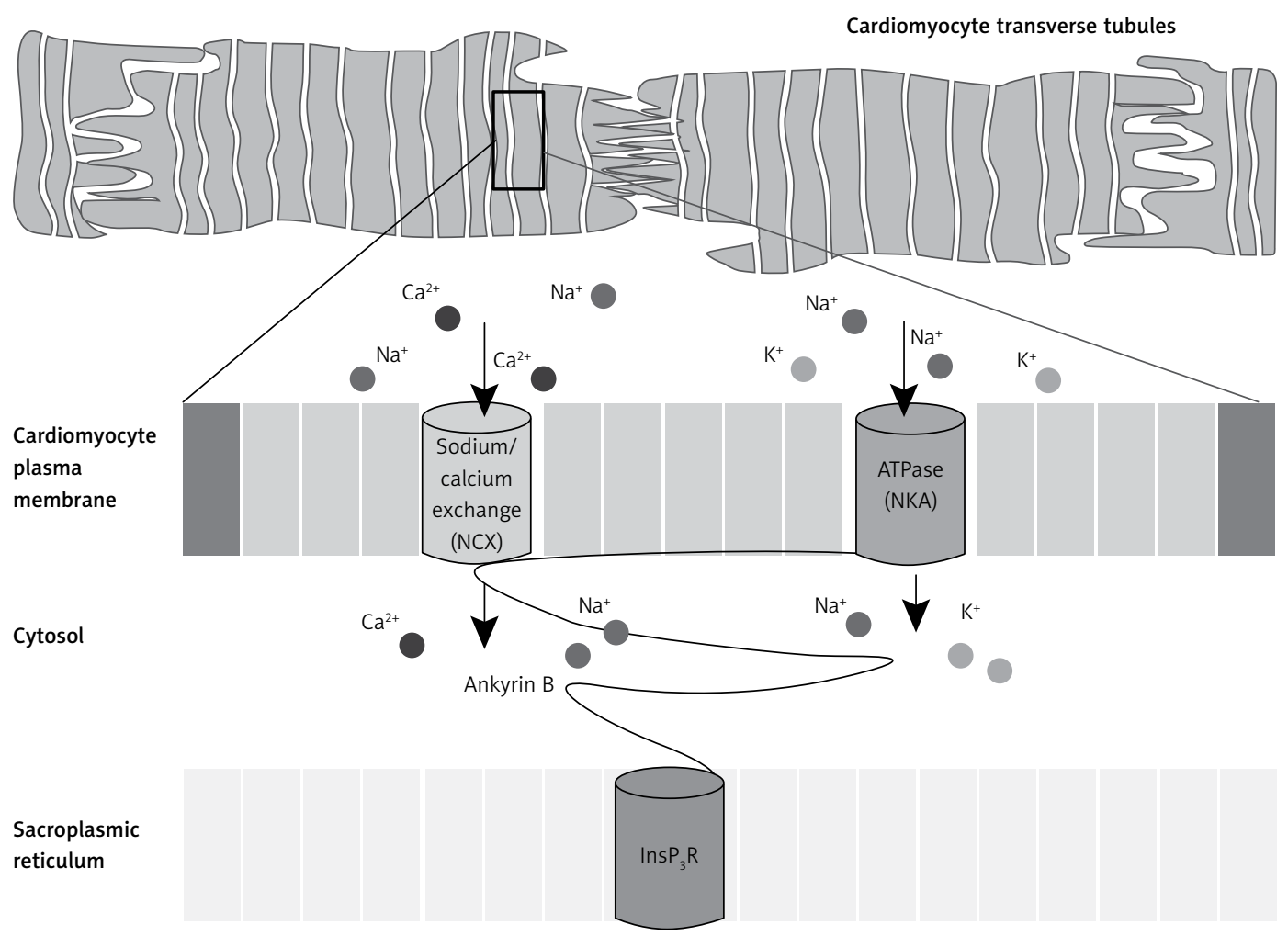

Figure 3. Targeting of ion channels and transporters at the sarcoplasmic reticulum and T-tubule junction membrane of the heart ventricles. The flux of calcium ions is facilitated by ankyrin B complex from the sarcoplasmic reticulum via inositol triphosphate receptors $\left(\operatorname{Ins}_{3} \mathrm{R}\right)$ and across the plasma membrane via the sodium calcium exchangers (NCX) and sodium potassium adenosine triphosphatase (NKA)

that ANK B is critical for $\beta$ II spectrin targeting in neonatal cardiomyocytes [26], whereas $\beta$ II spectrin is responsible for proper ANK B expression and localization in the mature cells [39]. It has also been shown that dysregulation in the ANK $B / \beta$ spectrin pathway is linked to congenital human heart disease $[39,40]$. Moreover, ANK B has been associated with the so-called corpus callosum hypoplasia, retardation, adducted thumbs, spastic paraplegia and hydrocephalus (collectively termed CRASH syndrome). Since the mutations of human L1 are located in the ANK binding site of the L1CAM proteins, it was shown that ANK B knocked out mice displayed loss of $\mathrm{L} 1$ from axons - a loss that has been directly linked with neurological symptoms including corpus callosum hypoplasia, spastic paraparesis [8], slurred speech, and mental retardation, together termed CRASH symptoms [ 1 , 28]. Evidence in animal studies shows that not only the interactions between L1 and ANK B are important but also the roles played by ANK B protein at the cellular level. The disturbance in the ANK B-mediated cellular events leads to neurological anomalies such as improper neurite growth and/or branching, axonal guidance and fasciculation [8].

In some cases, ANK B deficiency in mice has been linked with non-acute skeletal muscular phenotypes with elevated creatine kinase levels and occasionally disorganized sarcomeres. In addition, ANK B was recently found to interact with dystrophin, which plays a crucial role in skeletal muscle organization and integrity [10]. Similarly as in the case of ANK R, skeletal muscle disorders are yet to be reported in the case of ANK B dysfunction; nevertheless, their dysfunction may be compensated by ANK R and G co-expression [10]. However, at least two independent studies suggested that one of the muscular dystrophies (Becker's) interrupts ANK B/dystrophin interaction, resulting in inappropriate translocation of dystrophin in skeletal muscles [10, 41].

\section{Ankyrin G, bipolar disorder, schizophrenia and Brugada syndrome}

Encoded by the ank3 gene [11], located on human chromosome 10q21 [8], ANK G is a scaffold protein, which exists as different isoforms, ranging from a molecular weight ( $\mathrm{Mr}$ ) of 107 to $480 \mathrm{kDa}$ (Table I). The largest among all, ANK G protein is expressed in the epithelial tissues, kidneys, brain, lungs, cardiac and skeletal muscles [1, 12]. They have been implicated in a multitude of functions, such as neurogenesis and neuroprotection [15], as well as neuro-development and signalling [42]. Moreover, in an in vitro canine kidney cell line 
model it has been shown that $A N K B / \beta$ II spectrin domains are involved in lateral membrane formation [43-45]. Voltage gated sodium channels (VGSCs) have been suggested to be ANK G dependent and operate in their assembly at the axon initial segment (AIS) and nodes of Ranvier [46], where ank3 is thought to execute the localization of ion channels and GABAergic presynaptic knobs of the neurons [47]. Of all the ANK 3 isoforms, ANK $\mathrm{H}$ is required for the proper localization of voltage-gated Nav channels, KCNQ2/3 channels [48]. Not surprisingly, their dysfunction has been linked with several neurological disorders [11]. It should be noted that the genesis of the ank genes' mutations is still unclear. Nevertheless, several studies have suggested that ank3 polymorphisms lead to bipolar disorder (BD) [49] and schizophrenia. Bipolar disorder, also known as maniac-depressive syndrome, is a mental disorder characterized by episodes of mania, mood swings, and periods of depression. There is an environmental as well as genetic component to BD genesis [47]. Genome-wide association studies (GWAS) presented compelling evidence from compiled studies, using 1.8 million variants and having tested millions of single nucleotide polymorphisms [47] for differences between almost 4400 cases and a little over 6200 controls. They showed that variations in the ank3 and voltage-gated calcium channel subunit 1c (CACNA1C) are directly linked with the susceptibility to bipolar disorder [50]. In contradistinction some GWAS and targeted studies of ANK G failed to detect a significant correlation. Ank3 single nucleotide polymorphisms serve as markers of genetic variants related to disease that might be found in proximity to the gene [47]. Ankyrin $\mathrm{G}$ has been linked with a predisposition to anhedonia, a condition characterized by the lack of the capacity to feel pleasure and poorer cognition. Anhedonia is usually a characteristic feature of mental disorders such as schizophrenia and personality disorder [47]. Ank3 polymorphisms have also been associated with decreased white matter integrity [15]. The data above attest to the fact that variations in ank 3 contribute to physiological and functional alterations in the brain, which may be linked to BD. Moreover, the under-regulation of ANK G in the superior temporal gyrus of schizophrenia patients might serve as a novel biomarker for schizophrenia psychopathology [47]. Furthermore, the BD and schizophrenia groups (psychiatric genomics consortium) also provided significant associations (using over 16,000 cases and 14,000 control cases) between bipolar disorder and schizophrenia at the 5 prime region of the ank3, CACNA1C as well as in the chr3p21.3 locus. Although ANK G protein's mode of action contributing to BD and schizophrenia is still un- known, hopefully in the near future more studies of the neural circuitry of human brains and the roles played by ank3 therein will pave way for a much better understanding of the relationship between ANK G and mental illnesses [47].

All three ANK gene products have been observed in ventricular cardiomyocytes; however, the way they are conjointly expressed and scattered in the heart is still unclear [10]. Notwithstanding this, ANK $G$ has been observed to play pivotal roles in modulating the proper expression and membrane targeting of cardiac VGSCsNav1.5 [11] (Figure 4), and a dysfunction in the interactome of ANK/Nav1.5 is linked with a human cardiac arrhythmia, termed Brugada syndrome $[5,10]$. Voltage gated sodium channels (VGSCs) initiate rapid electrical signals (action potential), a requisite for the rhythmic beating of the heart. Not surprisingly, a mutation in the Nav1.5-sodium channel voltage gated type $\mathrm{V} \alpha$ subunit gene (SCN5A gene) has been demonstrated to disrupt their association with ANK G, resulting in Brugada syndrome, a cardiac anomaly characterized by ST segment elevation in precordial ECG leads, right bundle branch block, and susceptibility to fatal arrhythmia [8]. Ankyrin G and Nav1.5 are found at the intercalated disc and T-tubule membranes in cardiomyocytes, and they also co-immunoprecipitate from detergent-soluble lysates of heart $[8,10]$, attesting to the aforementioned association of ANK G/Nav1.5 and the function of ANK G in targeting Nav1.5 in excitable myocytes.

\section{Ankyrins repeat domain containing proteins}

As elaborated upon in the previous chapters, ANK are made up of three key domains: the $\mathrm{N}$-terminal containing ANK repeats, spectrin binding domain and the $C$-terminal regulatory domain. The former domain (ANK repeat motif) has been considered the most conserved amino acid motif [14] and is ubiquitously expressed in a variety of proteins in almost all life forms, including the viruses, archaea, and prokaryotes, and is most abundantly common in the eukaryotes [51]. The ANK repeat is a stretch of 33 amino acids [14] that mediate protein-protein interactions [51]. The major reason for ANK repeat containing proteins' diverse functions is their ability to interact with other polypeptides, principally with other ANK repeats. Some of these functions include modulation of the cell cycle, cell fate determination, regulation of transcription, cytoskeleton integrity [14] as well as structural and regulatory roles in the skeletal muscles [20]. In this chapter proteins containing ANK repeats (Table II) are expanded upon in terms of general structure, functions and how their dysfunction can lead to a diversity of health pertaining complications and diseases. 


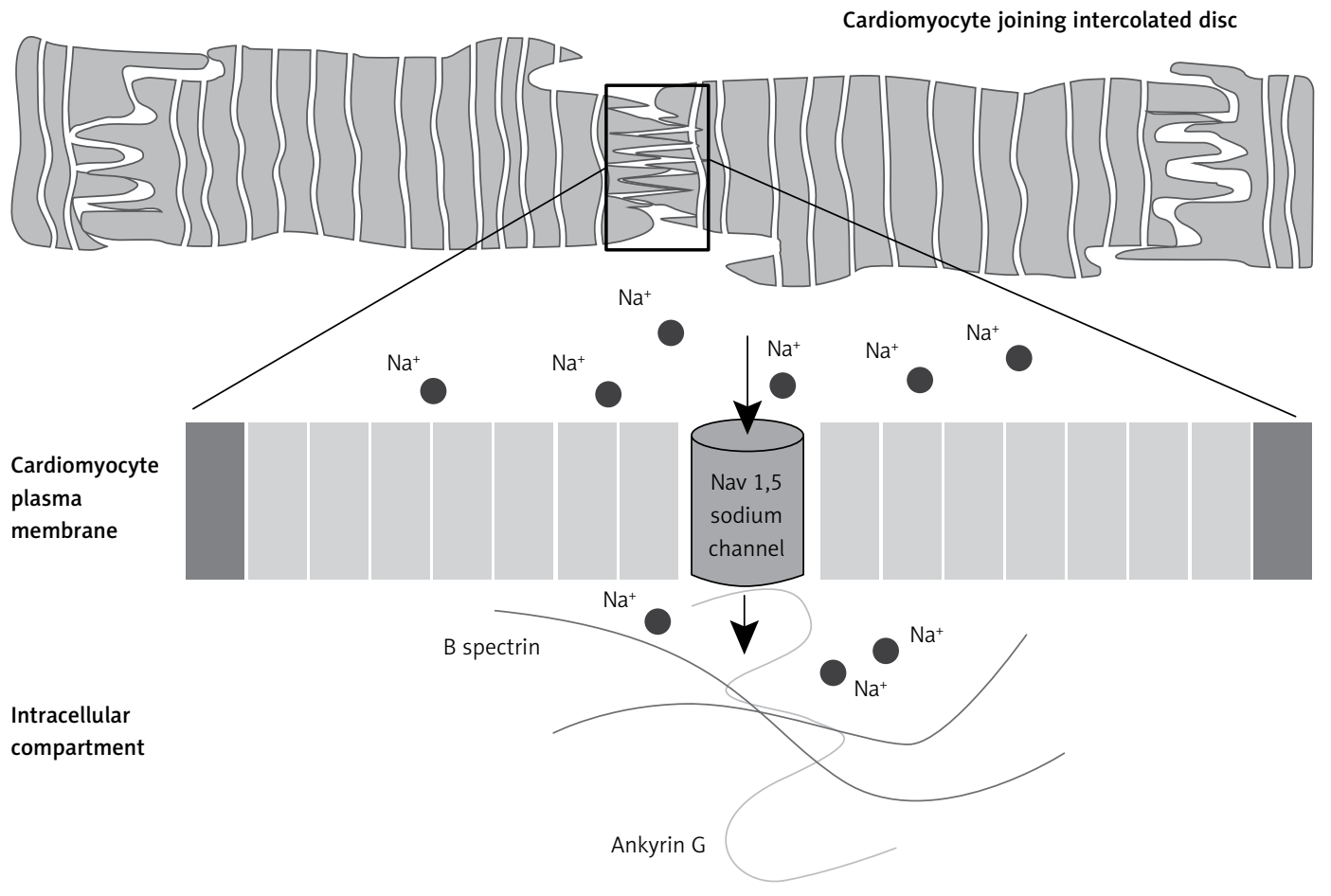

Figure 4. Association of ankyrin G/Nav1,5 cardiac sodium channel voltage gated type $\mathrm{V} \alpha$ subunit. Ankyrin $\mathrm{G}$ by interaction with B spectrin coordinates Nav1,5 cardiac sodium channel voltage gated type $V \alpha$ subunit and function of intercalated discs of cardiomyocyte in impulse propagation in the heart

\section{Muscle ankyrin repeat proteins in conjunction with muscle development and diseases}

Muscle ankyrin repeat proteins (MARPs) are a family of muscle proteins containing an ANK repeat domain [52]. They are located in the tissues of the heart and skeletal muscles and have been implicated in playing crucial structural and regulatory roles in the skeletal muscles $[52,53]$. Their exact role remains unclear. Barash et al., showed that different MARPs were up-regulated in mice after inducing eccentric contraction (EC) of their muscles, a process ensued by strengthening of the muscles and overall reduced susceptibility to further injuries [21]. However, the cellular basis for this response remains a subject for further exploration. Muscle ankyrin repeat proteins are also believed to play cofactor-like roles in local signalling pathways in muscle morphogenesis and cardiac hypertrophy. Before elucidating further on this topic, let us take a step back and review the different kinds of MARPs: CARP (Ankrd1) is a cardiac ankyrin repeat protein, which, as the name suggests, is predominantly expressed in the heart $[52,53]$, DARP (Ankrd23) is a diabetes-associated ankyrin repeat family of proteins, expressed in both the cardiac and skeletal tissues [14, 21, 52] and Ankrd2/Arpp-stands for ankyrin repeat domain 2/ankyrin repeat protein associated with PEST (proline, glutamic acid, serine and threonine) and proline-rich regions, which

Table II. The proteins containing ankyrin repeats and confirmed protein-protein interactions via their amino acid motif ([14], modified)

\begin{tabular}{|lccc|}
\hline Protein & ANK repeats & Role & Interacting proteins \\
\hline ANK & 24 & SR and synaptic membrane organization & Obscurin, spectrin \\
\hline Ankrd1/CARP & 4 & Stress response & YB-1 \\
\hline Ankrd2/Arpp & 4 & Stress response & Titin, myopalladin \\
\hline Ankrd23/DARP & 4 & Stress response and energy metabolism & Myopalladin, titin \\
\hline Notch & 7 & Muscle differentiation & SKIP \\
\hline
\end{tabular}

ANK - ankyrin, YB-1 - Y-box-binding protein 1, SKIP-ski-interacting protein. 
are similar to CARP, although unlike CARP they are more abundant in the skeletal muscles. Barash et al. hypothesized that the structural stability of skeletal muscles is attributable to the MARPs, as per their over-expression in the muscles following an EC, or as related to the gene expression response and remodelling of these muscles after injury [21]. This was demonstrated using mice models, whereby all of the three MARPs knocked out mice displayed a higher regenerative response to EC-induced muscle injury, suggesting the MARPs' modulatory role, as well as their function in the passive mechanical behaviour and structural stability of the tissue. This however does not imply that MARPs are essential for the basal functioning of skeletal muscles, as only insignificant effects were observed in muscle fibre size and distribution. Moreover, there has not yet been any report of over-expression or compensation by the remaining MARPs when one or more of the MARP genes are knocked out. This is clearly an understudied part of this research [21].

\section{Ankrd1/cardiac adriamycin-responsive protein}

Encoded by the ankrd1 gene, ankyrin repeat domain 1 proteins, also known as cardiac ankyrin repeat protein or cardiac adriamycin-responsive protein (CARP) [21, 53], are expressed highly in the heart [54], and endothelial tissues as well as scarcely in the skeletal muscles. They are characterized by the presence of four ANK repeat motifs in their central region [14]. They have been implicated in several important functions, some of which are: negative transcriptional regulation of cardiac gene expression in the heart of a fetus, myofibrillar assembly, physiological reshaping of ventricular myocardium, stretch sensing and relaying information between the sarcoplasm and the nucleus [53]. As testimony to their importance, not long ago Ankrd1/CARP proteins emerged as markers in congenital heart disease. In vivo functions of Ankrd1/CARP still remain elusive. This is mainly due to the failure to show either heart-specific deletion/ down-regulation of the ankrd1 gene or cardio-restricted ankrd1 trans-genesis. Nonetheless, in vivo over-expression of CARP in fetal, neonatal and adult heart in response to stress attests to their importance as regulators of physiological and pathological processes. Kojic et al. posited that an increase in load delivered to cardiac myocytes results in the activation of multiple signalling pathways that, in turn, activate gene expression as part of a hypertrophic response [55]. However, the mechanism by which muscle stretch is sensed and signals are transduced is still unknown. Moreover, Nakada et al. conducted an investigation using immunochemical techniques, analysing the expression of CARP protein in biopsied skeletal muscles of 11 and 14 cases of spinal muscular atrophy (SMA) and congenital myopathy, respectively. They found that CARP expression was up-regulated in atrophic myofibres of SMA1 and 2, while its expression in nemaline myopathy was found to be higher in severely damaged myofibres, suggesting a direct correlation between CARP's expression and severity of the disease [54]. In contradistinction, no CARP was found in central core disease (another subtype of myopathy). It is safe to suggest then that based on these findings and with more research, CARP could be the next novel biomarker in the diagnosis of SMA, congenital myopathy [54] and congenital heart disease [53]. Furthermore, not long ago, CARP was found to be induced in muscle regeneration in the muscular dystrophies and in skeletal muscle in amyotrophic lateral sclerosis (ALS) [54]. That is also another avenue to explore.

\section{Ankrd2/Arpp}

Encoded by the ankrd2 gene located in human chromosome region 10q23.31-23.32 [56], ankyrin repeat domain 2, also known as ankyrin repeat associated with PEST, is a family of proteins belonging to the MARP family that are similar to CARP as they are made up of nine exons, four of which are tandem ANK repeated motifs, as well as possessing an NF- $\kappa B$ (nuclear factor kappalight-chain-enhancer of activated $B$ cells) binding sequence indicating that ankrd2 gene expression may be regulated by NF- $\kappa B$, as this expression is usually induced by interleukin (IL)-1, tumour necrosis factor (TNF)- $\alpha$ and lipopolysaccharide (LPS). Unlike CARPs, they are abundantly expressed in the skeletal muscles [21, 55, 57], specifically the type I fibre $[21,56]$. They are also found in myocardium [57] as well as in kidneys in small amounts. The PEST sequence of Arpp is also of extreme importance due to its proteolytic signalling that results in rapid destruction of proteins. It has been observed that their expression is higher with denervation [21, 57] or stretching [55]. In mice studies, it was found that ankrd2 is usually induced in muscles as a stress response, suggesting possible involvement in the regulation of muscle hypertrophy [55]. It has also been observed to play a significant role in the stretch response associated with slow muscle movement. This is evident in muscle dystrophy cases, where ankrd 2 seems to be down-regulated. Moreover, it was observed that ankrd2 was up-regulated during muscle cell differentiation, suggesting its function in regulating gene expression during muscle development by interacting with both sarcoplasmic structural and nuclear proteins in the negative regulation of myocyte specialization [55]. 


\section{Ankrd23/DARP}

Ankrd23, also described as a diabetes-associated ankyrin repeat protein (DARP), is encoded by the ankrd23 gene and expressed in myocardium and skeletal muscles. Like its homologous cousin proteins of the MARP family, DARP also contains four tandem ANK repeats in its central region. Diabetes-associated ankyrin repeat proteins are found in the nucleus and play the role of transcriptional regulators. Diabetes-associated ankyrin repeat proteins have been found to be induced during recovery following a prolonged period of hunger. This suggests that they may participate in energy metabolism and muscle stress pathways [57]. Not surprisingly, in type 2 diabetes, a metabolic disorder characterized by hyperglycaemia with a relative lack of insulin in the pancreas and in insulin-resistant animals, they have been found to be over-expressed, suggesting them as useful biomarkers in the diagnosis of diabetes. Diabetesassociated ankyrin repeat proteins, like CARPs, are also localized in response to stress stretch signals, suggesting a molecular link between myofibrillar stretch-induced signalling pathways and muscle gene expression [58].

\section{Notch proteins}

Notch proteins belong to the trans-membrane proteins class, possessing several domains, including an epidermal growth factor (EGF) domain [59], an ANK repeat domain and a PEST domain. In humans there are four Notch receptors, Notch1Notch4, implicated in crucial functions, from cell fate modulation and regulation of homeostasis in the liver $[59,60]$ to inducing proliferation and differentiation. This is mostly ascribed to their evolutionarily conserved signalling pathway. Yap et al. demonstrated their oncogenic functions [59]. It was demonstrated that their levels were overly expressed in head and neck squamous cell carcinoma (HNSCC), suggesting their tumourigenic roles (as oncogenes). Recently the predictive value of Notch signalling in renal carcinoma patients has been suggested [61]. Their association with cancer was first demonstrated long before this in T-cell acute lymphoblastic leukaemia [59, 62]. A mutation in the notch 1 gene has been confirmed to be directly linked with HNSCC. In diseases such as Alagille syndrome (a genetic disorder affecting the liver, kidney and heart) [60], cerebral autosomal dominant arteriopathy with subcortical infarcts and leukoencephalopathy (CADASIL, a neurological disorder) [51] as well as in breast cancer, the Notch levels are also up-regulated. According to one of the theories, when Notch proteins are activated, they promote cell growth, angiogenesis and inhibition of apoptosis - the key processes which, when left unchecked, lead to formation of tumours or/and cancer. In contradistinction, the same study also revealed tumour suppressive roles of Notch, confirming the hypothesis that mutations in notch genes produce truncated proteins, which lead to loss-of-function phenotypes.

\section{Therapeutic applications of proteins containing ankyrin repeat domain}

In the previous chapters, diagnostic applications of ANK and ANK repeat domain containing proteins were discussed. It was seen how variations, mutations and/or dysfunction of the ANK genes can have potential health repercussions, resulting in diseases, and how the identification of these proteins, either down- or up-regulated, could serve as biomarkers for the given diseases. In this section, the therapeutic applications of proteins containing ANK repeats are elaborated upon.

\section{Designed ankyrin repeat proteins: engineered scaffold protein-drugs in therapy}

It has been extremely difficult to find an ideal drug, one that would have, among other features, high affinity and specificity to its targets, low cost of mass production, high solubility and stability, as well as the existence of assorted ways to deliver them to any part of the human body [63]. And even with the advent of antibodies and the success that they have had for over a century [56] in antibody-based therapy, they still face several drawbacks; they are difficult to compose, display low tissue penetration abilities [60], are too large to be administered via any route, require an expensive mammalian cell production system as they cannot be expressed in bacterial or yeast cell cultures $[56,63]$, and so forth. The emergence of recombinant genetically engineered proteins such as designed ankyrin repeat proteins (DARPins) seemed to have paved the way for a novel therapy with encouraging scientific success $[56,64]$ amidst the existing limitations. Designed ankyrin repeat proteins are a group of small engineered non-immunoglobulin scaffold proteins [65], the size of 14 to $21 \mathrm{kDa}$, derived from the ANK repeat proteins [64], usually containing between four and six of these ANK repeat motifs. It has been proposed that the higher the number of ANK repeats is, the more stable is the DARPin. This is also true for ANK proteins [51]. One of the advantages of using DARPins is that they exhibit high affinity (in as low as picomolar concentrations) and high specificity to the target protein as well as being thermodynamically stable $[66,67]$. The high specificity is ascribed to their rigid interface arising from 
three repeated $\beta$-turns in the ANK repeats (166 residues) [56]. Stumpp et al. described DARPins as being the most stable proteins to date with a melting point of over $100^{\circ} \mathrm{C}$ [64] and Binz et al. showed that recombinants possess a half-life of more than 60 days at $37^{\circ} \mathrm{C}$ [68]. Another advantage ascribed to their size and stability is the possibility of multiple routes of administration (topical, oral, nasal and inhaled). Depending on their design, their role ranges from receptor agonists or antagonists to enzyme inhibitors or target protein binders [65]. Furthermore, the absence of an Fc domain (effector function) ensures no direct link to the immune cells, which allows for high dosage implementation with minimal side effects [64]. Also, the lack of disulphide bonds in their structure enables their intra- and extracellular use [65]. Moreover, their miniature size allows for higher tissue penetration, a mechanism that makes targeting/neutralization (an antagonistic action) of foreign substances (ligands, cytokines, toxins) much easier even outside the blood circulation $[64,69]$. Furthermore, their potential to inhibit enzymes such as proteases, kinases and membrane proteins allosterically is vital for proper regulation and function of the body. Hanenberg et al. demonstrated using mouse models DARPins' therapeutic potential in the treatment of Alzheimer's, a neurodegenerative disease characterized by short-term memory loss and even dementia [66]. Amyloid- $\beta$ peptide (AB) - a crucial molecule involved in the inception and progression of Alzheimer's - was observed to be delayed from aggregating by binding specifically to DARPins and eventually neutralized and, ergo, prevented $A \beta$-mediated neurotoxicity in vitro. More than a dozen anti-amyloid immunotherapy clinical trials have been conducted to date for the treatment of Alzheimer's. Designed ankyrin repeat proteins might just be the answer to the question how we can fight diseases like Alzheimer's. Additionally, DARPins have been shown to play important roles in the pathological diagnosis and breast cancer therapy. Theurillat et al. presented evidence showing that DARPins perform robustly in binding to human epidermal growth factor receptor 2 (HER2), a protein that is over-expressed in some breast carcinomas. Therefore, by identifying the amplification status of the HER2 proto-oncogene using DARPins, this may underpin the successful diagnosis of breast cancer as well as indicating therapeutic possibilities [70].

In addition, DARPins were shown to block the entry of human immunodeficiency virus (HIV) into the cells by competing with the viral protein for the CD4 binding site on lymphocytes [63]. It is very likely that the fusion of different DARPINs would lead to the creation of one polyvalent molecule that expresses various functions. Simeon and Chen showed that the multi-DARPIN protein neutralizes the activity of vascular and hepatocyte growth factors, binds serum albumin and in a clinical trial was found to be safe [71].

Although DARPins are yet to be tested in clinical trials, their safety and efficacy have been shown in several preclinical models. Nevertheless, these experiments cannot fully rule out safety issues, such as potential immunogenicity in man [64] As already mentioned, DARPins are highly stable, have non-aggregation tendencies and lack an Fc domain. This is thought to reduce immunogenicity. Similarly, the abundance of ANK protein fragments in the red blood cells is thought to induce immunological tolerance, hence rendering them potentially non-immunogenic.

\section{Conclusions}

The abundance and universality of the ANK repeat motif in proteins should not be underestimated. As a signature amino acid motif, the ANK repeat domain is highly conserved in many proteins, in almost all life forms. Their conservation probably arose out of an evolutionary necessity, via variations and natural selection. The prevalence of human diseases that ensued from the disruption of ANK repeat domain containing proteins attests to their significance for normal physiology and function of the body. In the prokaryotes, for instance, they are secreted into the cytoplasm of the host, where they seem to exert epigenetic control over the hosts' gene expression as a mechanism to ensure the pathogen's survival [51]. More studies should be conducted to elaborate the pathogenicity that arises due to these protein domains. Additionally, the interactome (that is, the whole set of interactions) between ANK and other proteins in the cells is understudied and needs to be further explored for a better grasp of ANK functions [65, 72]. It is also worthwhile echoing the emergence of DARPins as blockbuster drugs in diagnosis and therapy [56, $66,67,70,73]$. These designed ANK repeat proteins, combined with the evolving knowledge in biotechnology and nanotechnology, could pave the way for DARPins as new frontier drugs in combating the ever increasing health concerns and emergence of diseases.

\section{Acknowledgments}

The authors thank Ms. Jolanta Cwynar for performing the linguistic correction.

\section{Conflict of interest}

The authors declare no conflict of interest. 


\section{References}

1. Bennett $\mathrm{V}$, Healy J. Organizing the fluid membrane bilayer: diseases linked to spectrin and ankyrin. Trends $\mathrm{Mol}$ Med 2008; 14: 28-36.

2. Chen DY, Fabrizio JA, Wilkins SE, et al. Ankyrin repeat proteins of Orf virus influence the cellular hypoxia response pathway. I Virol 2016; 91: e01430-16.

3. Al-Khodor S, Price CT, Kalia A, Abu Kwaik Y. Ankyrinrepeat containing proteins of microbes: a conserved structure with functional diversity. Trends Microbiol 2010, 18: 132-9.

4. Ipsaro JJ, Huang L, Mondragón A. Structures of the spectrin-ankyrin interaction binding domains. Blood 2009; 113: 5385-93.

5. Cunha SR, Le Scouarnec S, Schott JJ, Mohler PJ. Exon organization and novel alternative splicing of the human ANK2 gene: implications for cardiac function and human cardiac disease. J Mol Cell Cardiol 2008; 45: 724-34.

6. Abriel H. Cardiac sodium channel Nav1.5 and interacting proteins: physiology and pathophysiology. J Mol Cell Cardiol 2010; 48: 2-11.

7. Cunha SR, Mohler PJ. Ankyrin-based cellular pathways for cardiac ion channel and transporter targeting and regulation. Semin Cell Dev Biol 2011; 22: 166-70.

8. Cunha SR, Mohler PJ. Ankyrin protein networks in membrane formation and stabilization. J Cell Mol Med 2009; 13: 4364-76.

9. Clarkson YL, Perkins EM, Cairncross CJ, et al. Beta-III spectrinunderpins ankyrin $\mathrm{R}$ function in purkinje cell dendritic trees: protein complex critical for sodium channel activity is impaired by SCA5-associated mutations. Hum Mol Genet 2014; 23: 3875-82.

10. Hashemi SM, Hund TJ, Mohler PJ. Cardiac ankyrins in health and disease. J Mol Cell Cardiol 2009; 47: 203-20.

11. Iqbal Z, Vandeweyer $G$, Van der voet $M$, et al. Homozygous and heterozygous disruptions of ANK3: at the crossroads of neurodevelopmental and psychiatric disorders. Hum Mol Genet 2013; 22: 1960-70.

12. Baines AJ. The spectrin-ankyrin-4.1-adducin membrane skeleton: adapting eukaryotic cells to the demands of animal life. Protoplasma 2010; 244: 99-131.

13. Hsu WCJ, Nilsson CL, Laezza F. Role of the axonal initial segment in psychiatric disorders: function, dysfunction, and intervention. Front Psychiatry 2014; 5: 109.

14. Tee JM, Peppelenbosch MP. Anchoring skeletal muscle development and disease: the role of ankyrin repeat domain containing proteins in muscle physiology. Crit Rev Biochem Mol Biol 2010; 45: 318-30.

15. Kloiber S, Czamara D, Karbalai N, et al. ANK3 and CACNA1C - missing genetic link for bipolar disorder and major depressive disorder in two German case-control samples. J Psychiatr Res 2012; 46: 973-9.

16. Nelson AD, Jenkins PM. Axonal membranes and their domains: assembly and function of the axon initial segment and node of Ranvier. Front Cell Neurosci 2017; 11: 136.

17. Schiffer JM, Malmstrom RD, Parnell J, et al. Model of the ankyrin and SOCS Box protein, ASB9, E3 ligase reveals a mechanism for dynamic ubiqutin transfer. Structure 2016; 24: 1248-56.

18. El Rafaey M, Mohler PJ. Ankyrins and spectrins in cardiovascular biology and diseases. Front Physiol 2017; 8: 852.

19. Mohler PJ, Rivolta I, Napolitano C, et al. Nav 1.5 E1053K mutation causing Brugada syndrome blocks binding to ankyrin-G and expression of Nav 1.5 on the surface of cardiomyocytes. Proc Natl Acad Sci USA 2004; 101: 17533-8.
20. Kojic S, Radojkovic D, Faulkner G. Muscle ankyrin repeat proteins: their role in striated muscle function in health and disease. Crit Rev Clin Lab Sci 2015; 48: 269-94.

21. Barash IA, Bang ML, Mathew L, Greaser ML, Chen J, Lieber RL. Structural and regulatory roles of muscle ankyrin repeat protein family in skeletal muscle. Am J Cell Physiol 2007; 293: 18-27.

22. Islam Z, Nagampalli RSK, Fatima MT, Ashrof GM. New paradigm in ankyrin repeats: beyond protein-protein interaction module. Int J Biol Macromol 2018; 109: 1164-73.

23. Kim DH, Park MJ, Gwon GH, et al. An ankyrin repeat domain of AKR2 drives chloroplast targeting through coincident binding of two chloropast lipids. Dev Cell 2014; 30: 598-609.

24. Woodford CR, Thoden JB, Holden HM. New role for the ankyrin repeat revealed by a study of the $\mathrm{N}$-formyltransferase from Providencia alcaligenes. Biochemistry 2015; 54: 631-8.

25. Davies JQ, Mc Laughlin T, Bennett V. Ankyrin-binding proteins related to nervous system cell adhesion molecules: candidates to provide transmembrane and intercellular connetions in adult brain. J Cell Biol 1993; 121: 121-33.

26. Mohler PJ, Yoon W, Bennett V. Ankyrin-B targets beta 2-spectrin to an intracellular compartment in neonatal cardiomyocytes. J Biol Chem 2004; 279: 40185-93.

27. Li J, Kline CF, Hund TJ, Anderson ME, Mohler PJ. Ankyrin $B$ regulates Kir 6.2 membrane expression and function in heart. J Biol Chem 2010; 285: 28723-30.

28. Hortsch M, Nagaraj K, Godenschwege TA. The interaction of L1-type proteins and ankyrins - a master swich for L1-type CAM function. Cell Mol Biol Lett 2009; 14: 57-69.

29. Perrotta S, Gallagher PG, Mohandas N. Hereditary spherocytosis. Lancet 2008; 372: 1411-26.

30. Xiaoqiu W, Bingmu F, Jinhong J, et al. A case report of hereditary spherocytosis with concomitant chronic myelocytic leukemia. Open Med 2016; 11: 152-4.

31. Hogas S, Bilha SC, Branisteanu D, et al. Potential novel biomarkers of cardiovascular dysfunction and disease: cardiolipin-1, adipokines and galectin-3. Arch Med Sci 2017; 13: 897-913.

32. Faridi KF, Lupton JR, Martin SS, et al. Vitamin D deficiency and non-lipid biomarkers of cardiovascular risk. Arch Med Sci 2017; 13: 732-7.

33. Michalski B, Szymczyk E, Peczek L, et al. The role of selected adipokines and ghrelin in the prognosis after myocardial infarction in a 12-month follow-up in the presence of metabolic syndrome. Arch Med Sci 2017; 13: 785-94.

34. Soran H, Adam S, Mohammad JB, et al. Hypercholesterolaemia - practical information for non-specialist. Arch Med Sci 2018; 14: 1-21.

35. Stulc T, Lanska V, Snejdrlova M, Vrablik M, Prosikova M, Ceska R. A comprehensive guidelines-based approach reduces cardiovascular risk in everyday practice: the VARO study. Arch Med Sci 2017; 13: 705-10.

36. Choy AM, Darbar D, Dell'Orto S, Roden DM. Exagerrated QT prolongation after cardioversion of atrial fibrillation. J Am Coll Cardiol 1999; 34: 396-401.

37. Tomaselli GF. A failure to adapt: ankyrins in congenital and acquired arrythmias. Circulation 2007; 115: 428-9.

38. Zienciuk A, Szwoch M, Raczak G. Atrial fibrillation in the long QT syndrome. Kardiol Pol 2009; 67: 681-4.

39. Smith SA, Sturm AC, Curran J, et al. Dysfunction the beta II spectrin-dependent cytoskeleton underlies human arrhythmia. Circulation 2015; 131: 695-708.

40. Derbala MH, Guo AS, Mohler PJ, Smith SA. The role of betall spectrin in cardiac health and disease. Life Sci 2018; 192: 278-85. 
41. Ayalon G, Davis JQ, Scotland PB, Bennett V. An ankyrinbased mechanism for functional organization of dystrophin and dystroglycan. Cell 2008; 135: 1189-200.

42. Paez-Gonzalez P, Abdi K, Luciano D, et al. Ank3-dependent SVZ niche assembly is required for the continued production of new neurons. Neuron 2011; 71: 61-75.

43. He $M$, Jenkins P, Bennett V. Cysteine 70 of ankyrin-G is S-palmitoylated and is required for function of ankyrin- $G$ in membrane domain assembly. J Biol Chem 2012; 287: 43995-4005.

44. He M, Abdi KM, Bennett V. Ankyrin-G palmitoylation and beta II-spectrin binding to phosphoinositide lipids drive lateral membrane assembly. J Cell Biol 2014; 206: 273-88.

45. Jenkins PM, He M, Bennett V. Dynamic spectrin/ankyrin-G microdomains promote lateral membrane assembly by opposing endocytosis. Sci Adv 2015; 1: e1500301.

46. Gasser A, Ho TSY, Cheng X, et al. An ankyrin G-binding motif is necessary and sufficient for targeting Nav1.6 sodium channels to axon initial segments and nodes of Ranvier. J Neurosci 2012; 32: 7232-43.

47. Leussis MP, Madison JM, Petryshen TL. Ankyrin 3: genetic association with bipolar disorder and relevance, to disease pathophysiology. Biol Mood Anxiety Disord 2012; 2: 18.

48. Jenkins PM, Kim N, Jones SL, et al. Ankyrin-G: a critical innovation in vertebrate evolution of fast and integrating neuronal signaling. Proc Natl Acad Aci USA 2015; 112: 957-64.

49. Quinn EM, Hill M, Anney R, Gill M, Corvin AP, Morris DW. Evidence for cis-acting regulation of ANK3 and CACNA1C gene expression. Bipolar Disord 2010; 12: 440-5.

50. Ferreira MA, Donovan MC, Meng YA, et al. Collaborative genome-wide association analysis supports a role for ANK3 and CACNA1C in bipolar disorder. Nat Genet 2008; 40: 1056-8

51. Jernigan KK, Bordenstein SR. Ankyrin domains across the Tree of Life. Peer J 2014; 2: e264.

52. Bang ML, Gu Y, Dalton ND, Peterson KL, Chien KR, Chen J. The muscle ankyrin repeat proteins CARP, ankrd2, and DARP are not essential for normal cardiac development and function at basal conditions and in response to pressure overload. PLoS One 2014; 9: e93638.

53. Mikhailov AT, Torrado $M$. The enigmatic role of the ankyrin repeat domain 1 gene in heart development and disease. Int J Dev Biol 2008; 52: 811-21.

54. Nakada C, Oka A, Nonaka I, et al. Cardiac ankyrin repeat protein is preferentially induced in atrophic myofibers of congenital myopathy and spinal muscular atrophy. Pathol Int 2003; 53: 653-8.

55. Kojic S, Medeot E, Guccione E, et al. The Ankrd2 protein, a link between the sarcomere and the nucleus in skeletal muscle. J Mol Biol 2004; 339: 313-25.

56. Gebauer M, Skerra A. Engineered protein scaffolds as next-generation antibody therapeutics. Curr Opin Chem Biol 2009; 13: 245-55.

57. Miller MK, Bang ML, Witt CC, et al. The muscle ankyrin repeat proteins: CARP, ankrd2/Arpp and DARP as a family of titin filament-based stress response molecules. Mol Biol 2003; 333: 951-64.

58. Ishiguro N, Motoi T, Araki N, Ito H, Moriyama M, Yoshida H. Expression of cardiac ankyrin repeat protein, CARP, in malignant tumors: diagnostic use of CARP protein immunostaining in rhabdomyosarcoma. Hum Pathol 2008; 39: 1673-9.

59. Yap L, Lee D, Khairuddin A, et al. The opposing roles of NOTCH signalling in head and neck cancer: a mini review. Oral Dis 2015; 21 : 850-7.
60. Geisler F, Strazzabosco M. Emerging roles of Notch sig naling in liver disease. Hepatology 2015; 61: 382-92.

61. Jędraszka D, Orzechowska M, Bednarek AK. Predictive values of Notch signalling in renal carcinoma. Arch Med Sci 2017; 13: 1249-54.

62. Lin JT, Wu MS, Wang WS, et al. All-trans retinoid acid increases Notch1 transcript expression in acute promyelocytic leukemia. Adv Ther 2015; 20: 337-43.

63. Tomlinson IM. Next-generation protein drugs. Nat Biotechnol 2004; 22: 521-2.

64. Stumpp MT, Binz HK, Amstutz P. DARPins: a new generation of protein therapeutics. Drug Discov Today 2008; 13: 695-701.

65. Bennett V, Lorenzo DN. An adaptable spectrin/ankyrinbased mechanisms for long-range organization of plasma membranes in vertebrate tissues. Curr Top Membr 2016; 77: 143-84.

66. Hanenberg M, McAfoose J, Kulic L, et al. Amyloid-ßeta peptide-specific darpins as a novel class of potential therapeutics for alzheimer disease. J Biol Chem 2014; 289: 27080-9.

67. Steiner D, Forrer P, Plückthun A. Efficient selection of DARPins with sub-nanomolar affinities using SRP phage display. J Mol Biol 2008; 382: 1211-27.

68. Binz HK, Stumpp MT, Forrer P, Amstutz P, Pluckthum A Designing repeat proteins: well-expressed, soluble and stable proteins from combinational libraries of consensus ankyrin repeat proteins. J Mol Biol 2003; 332: 489 . 503.

69. Pluckthun A. Designed ankyrin repeat proteins (DARPINS): binding proteins for research, diagnostics, and therapy. Annu Rev Pharmacol Toxicol 2015; 55: 489-511.

70. Theurillat JP, Dreier B, Nagy-Davidescu G, et al. Designed ankyrin repeat proteins: a novel tool for testing epidermal growth factor receptor 2 expression in breast cancer. Mod Pathol 2010; 23: 1289-97.

71. Simeon R, Chen Z. In vitro-engineered non-antibody protein therapeutics. Protein Cell 2018; 9: 3-14.

72. Swayne LA, Murphy NP, Asuri S, et al. Novel variant in the ANK2 membrane-binding domain is associated with ankyrin-B syndrome and structural heart disease in a first nations population with a high rate of long QT syndrome. Circ Cardiovasc Genet 2017; 10: e001537.

73. Nuttall SD, Walsh RB. Display scaffolds: protein engineering for novel therapeutics. Curr Opin Pharmacol 2008; 8: 609-15. 